\title{
(2) Association of physical activity with risk of OPEN ACCESS hepatobiliary diseases in China: a prospective cohort
study of 0.5 million people
}

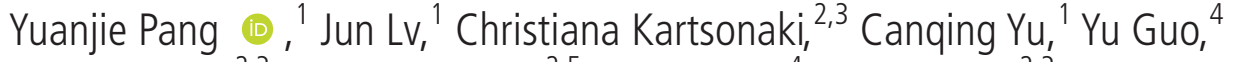 \\ Huaidong Du, ${ }^{2,3}$ Derrick Bennett, ${ }^{2,5}$ Zheng Bian, ${ }_{1}^{4}$ Yiping Chen, ${ }^{2,3}$ Ling Yang, ${ }^{2,3}$ \\ lain Turnbull, ${ }^{2}$ Hao Wang, ${ }^{6}$ Hui Li, ${ }^{7}$ Michael V Holmes, ${ }^{2,3,5}$ Junshi Chen, ${ }^{8}$ \\ Zhengming Chen, ${ }^{2,3}$ Liming $\mathrm{Li}^{1}$
}

\begin{abstract}
- Additional material is published online only. To view please visit the journal online (http://dx.doi.org/10.1136/ bjsports-2020-102174).
\end{abstract}

For numbered affiliations see end of article.

\section{Correspondence to} Professor Zhengming Chen; zhengming.chen@ndph.ox. ac.uk

Professor Liming Li; Imleeph@vip.163.com

Accepted 7 July 2020
Check for updates

(c) Author(s) (or their employer(s)) 2020. Re-use permitted under CC BY. Published by BMJ.

To cite: Pang Y, LV J, Kartsonaki C, et al. Br J Sports Med Epub ahead of print: [please include Day Month Year]. doi:10.1136/ bjsports-2020-102174

\section{ABSTRACT}

Objective There is limited prospective evidence on the association of physical activity with hepatobiliary cancer subtypes and other major hepatobiliary diseases, especially in China. We aimed to quantify the associations with risk of these diseases.

Methods The study population involved 460937 participants of the prospective China Kadoorie Biobank aged 30-79 years from 10 diverse areas in China without history of cancer or hepatobiliary disease at baseline. Cox regression was used to estimate adjusted hazard ratios (HRs) for each disease associated with self-reported total and domain-specific physical activity (occupational and non-occupational, ie, leisure time, household and commuting).

Results During 10 years of follow-up, 22012 incident cases of hepatobiliary diseases were recorded. The overall mean (SD) total physical activity was 21.2 (13.9) metabolic equivalent of task (MET)-hours/day, with $62 \%$ from occupational activity. Total physical activity was inversely associated with hospitalised non-alcoholic fatty liver disease (HR comparing top vs bottom quintile: 0.62, $95 \%$ confidence interval (CI) 0.53 to 0.72$)$, viral hepatitis $(0.73,95 \% \mathrm{Cl} 0.62$ to 0.87$)$, cirrhosis $(0.76,95 \% \mathrm{Cl}$ 0.66 to 0.88$)$ and liver cancer $(0.81,95 \% \mathrm{Cl} 0.71$ to $0.93)$, as well as gallstone disease $(0.86,95 \% \mathrm{Cl} 0.81$ to 0.90$)$, gallbladder cancer $(0.51,95 \% \mathrm{Cl} 0.32$ to 0.80$)$ and biliary tract cancer $(0.55,95 \% \mathrm{Cl} 0.38$ to 0.78$)$. The associations for occupational physical activity were similar to those for total physical activity, but for nonoccupational physical activity they differed by disease subtype. For leisure-time physical activity, there was an inverse association with liver cancer and an inverse trend for gallstone disease (HR comparing $\geq 7.5 \mathrm{MET}$-hours/day with none: $0.83,95 \% \mathrm{Cl} 0.75$ to 0.91 and $0.82,95 \% \mathrm{Cl}$ 0.66 to 1.01 )

Conclusion Among Chinese adults, high total physical activity, particularly occupational physical activity, was inversely associated with risk of major hepatobiliary cancers and diseases, including non-alcoholic fatty liver disease, cirrhosis and certain types of cancer.

\section{INTRODUCTION}

Hepatobiliary disease is a major cause of mortality and morbidity and accounted for 2.4 million deaths globally in $2017 .{ }^{1}$ Worldwide, about half of deaths from liver cancer and 15\% from cirrhosis occur in China. ${ }^{1}$ In recent decades, the age-standardised mortality rates from liver cancer and cirrhosis have been declining in China due to elimination of aflatoxin and control of hepatitis B virus (HBV), ${ }^{2}$ whereas the incidence rate of non-alcoholic fatty liver disease has been rising due to increasing prevalence of a sedentary lifestyle and Westernised diets. ${ }^{3}$ The most common type of gallbladder disease is gallstones, affecting 10\%-20\% of the global adult population. ${ }^{45}$ East Asian countries, including China, have been traditionally considered as areas with low risk of gallstones. ${ }^{4}$ Gallbladder and biliary tract cancer (GBTC) is a rare cancer, and its incidence mirrors that of gallstones, suggesting a similar risk factor profile for both diseases. ${ }^{1}$ Gallbladder disease and cancer may increase in China due to increasing prevalence of lifestyle-related risk factors, particularly obesity and physical inactivity.

Possible lifestyle-related risk factors for hepatobiliary diseases include smoking, alcohol and dietary factors, as well as metabolic risk factors including general and central adiposity, low leisure-time physical activity and diabetes. ${ }^{6-10}$ For physical activity, prospective studies have shown inverse associations of total and leisure-time physical activity with liver cancer, non-alcoholic fatty liver disease and gallbladder disease, ${ }^{11-15}$ but there is limited evidence on other hepatobiliary cancers and diseases. Hepatobiliary diseases, including chronic liver disease and gallbladder disease, lie on the pathophysiological pathway to hepatobiliary cancers and may share metabolic risk factors related to the insulin resistance syndrome. ${ }^{5-816}$ As insulin resistance has been hypothesised as a possible mechanism underlying the association of physical activity with hepatobiliary cancer, ${ }^{17}$ assessing the associations of physical activity with non-cancer outcomes may provide valuable insights into disease aetiology and inform prevention. Moreover, the majority of previous studies were in Western populations, where the patterns of metabolic risk factors, physical activity and diseases differ from those in China. For example, HBV accounts for $45 \%$ of liver cancer and cirrhosis in China, ${ }^{2}$ whereas alcohol accounts for $30 \%$ of liver cancer ${ }^{18}$ and $30 \%-40 \%$ of cirrhosis in Western countries. ${ }^{19} 20$ Occupational physical activity is the largest domain in China, ${ }^{21}$ whereas leisure-time physical activity is the largest domain in Western populations. ${ }^{22}$ However, there is limited evidence on domain-specific physical activity and 
hepatobiliary diseases both in China and in Western countries. Reliable assessment of the associations of physical activity with many different hepatobiliary diseases in Chinese may help inform disease prevention and better understanding of aetiology.

Our aims were to (1) quantify the associations of total, occupational and non-occupational physical activity with the risk of hepatobiliary diseases; (2) examine whether these associations differ by region, sex, age, sedentary leisure time, work activity intensity and different levels of baseline risk factors; and (3) assess whether additional adjustment for metabolic risk factors (eg, adiposity and diabetes) could alter the associations of physical activity with hepatobiliary diseases. We hypothesised that (1) total and occupational physical activity was associated with lower risk of hepatobiliary diseases; and (2) the associations of non-occupational physical activity with hepatobiliary diseases differed by domain of physical activity due to the different nature of activity in Chinese compared with Western populations (eg, unique patterns of leisure-time physical activity, urban-rural differences).

\section{METHODS}

\section{Study population}

The study recruited 512715 participants aged 30-79 years from 10 geographically defined localities (five urban and five rural) in China during 2004-2008. ${ }^{23}$ The study areas were selected to provide diversity in risk exposure and disease patterns, while taking into account population stability, quality of mortality and morbidity registries, capacity, and long-term commitment within the areas. At local study assessment clinics, participants completed an interviewer-administered, laptop-based questionnaire on sociodemographic characteristics, lifestyle factors, personal and family medical history, and current medication. A range of physical measurements were recorded by trained technicians, including height, weight, hip and waist circumference, bioimpedance, lung function, blood pressure, and heart rate, using calibrated instruments with standard protocols. All participants provided a $10 \mathrm{~mL}$ non-fasting blood sample for immediate on-site test of random plasma glucose (SureStep Plus metre; LifeScan, Johnson \& Johnson) and HBsAg (ACON Biotech).

\section{Assessment of physical activity}

The questions on physical activity and sedentary leisure time were adapted from validated questionnaires used in several other studies, including the European Prospective Investigation into Cancer and Nutrition (EPIC) and the Shanghai Women's Health Study, ${ }^{24} 25$ with some additional modifications after a China Kadoorie Biobank (CKB) pilot study. At baseline and subsequent resurveys, participants were asked about the frequency, duration and type (intensity) of physical activity in four domains (ie, occupation, commuting, housework and leisure-time exercise) during the past 12 months. To quantify the amount of physical activity, metabolic equivalent of tasks (MET) from the 2011 update of a compendium of physical activities was used. ${ }^{26}$ The MET value for a particular type of physical activity represents the ratio of the energy expended per kilogram of body weight per hour during that activity relative to that expended when sitting quietly. The number of hours spent per day participating in each activity was multiplied by the MET value for that activity, and the daily amount of total physical activity was obtained by summing the MET-hours/day for activities related to occupational and nonoccupational (ie, commuting, housework and non-sedentary leisure time) activities. Hours spent per day on sedentary leisuretime activities (such as television watching, reading, and playing cards or mahjong) and sleeping were also recorded, but were not included in the physical activity calculation. Online supplementary table 1 shows the types, MET values, codes and intensity categories of physical activity.

\section{Follow-up for morbidity and mortality}

The vital status of each participant was determined periodically through the China Center for Disease Control and Prevention's Disease Surveillance Points system and national health insurance system, ${ }^{27}$ supplemented by regular checks against local residential and administrative records and by annual active confirmation through street committees or village administrators. Additional information about major diseases and any episodes of hospitalisation was collected through linkages, via each participant's unique national identification number, with disease registries (for cancer, ischaemic heart disease, stroke and diabetes) and national health insurance claims databases, which have almost universal coverage in the study areas. All events were coded using the International Classification of Diseases, 10th Revision (ICD-10) by trained staff who were blinded to baseline information and reviewed centrally for consistency. ${ }^{23}$ The classification and distribution of hepatobiliary diseases are shown in online supplementary table 2. By 1 January 2017 (censoring date for the present analyses), $44066(8.6 \%)$ participants had died and $4751(1.0 \%)$ were lost to follow-up.

\section{Statistical analysis}

We excluded individuals with history of cancer $(n=2578)$, cirrhosis or hepatitis $(n=6139)$, a positive HBsAg test $(n=17$ 170), or gallbladder disease at baseline $(n=25891)$, leaving 460937 individuals for the main analysis. Mean values and prevalence of baseline characteristics were calculated for categories of total physical activity at baseline, standardised to age (in 5-year groups), sex and area structure of the CKB population.

Cox proportional hazards models with age as the underlying time scale and delayed entry at age at baseline were used to estimate HRs of specific disease incidence associated with physical activity levels. Models were stratified by sex and study area, and adjusted for age at baseline, education, household income, smoking, alcohol, self-rated health, diabetes, cardiovascular disease, respiratory disease, rheumatoid arthritis and sedentary leisure time. Physical activity was categorised by splitting at quintiles in order to assess the shape of the association. If the association was linear, then physical activity was also modelled as a continuous variable to estimate the risk associated with a 4 MET-hours/day higher level of physical activity. Separate analyses were conducted for occupational and non-occupational physical activity with mutual adjustment. For exposure variables with more than two categories, all HRs are presented with 'floating' standard errors (SEs) to facilitate comparisons between groups. ${ }^{28}$ The CKB estimates for physical activity and hepatobiliary cancers were meta-analysed with estimates from published prospective cohort studies using a random-effects meta-analysis. Details of the study selection are reported in online supplementary figure 1. Regression dilution was corrected for using the McMahon-Peto method (online supplementary table 3). ${ }^{29-32}$ Further details of the statistical analysis including covariates, regression dilution and sensitivity analyses are reported in online supplementary eMethods. Statistical analysis was done using R 2.14.2. 
Table 1 Baseline characteristics of participants by level of total physical activity

\begin{tabular}{|c|c|c|c|c|c|}
\hline \multirow[b]{3}{*}{ Variable* } & \multicolumn{5}{|c|}{ Total physical activity (MET-hours/day) } \\
\hline & $0-8.9$ & $8.9-14.3$ & $14.3-22.0$ & $22.0-33.4$ & $\geq 33.4$ \\
\hline & $(n=91228)$ & $(n=91110)$ & $(n=92604)$ & $(n=92655)$ & $(n=93340)$ \\
\hline Age (SD), years & $58.4(10.9)$ & $54.7(10.6)$ & $50.5(10.0)$ & $48.5(9.2)$ & $47.1(8.2)$ \\
\hline Female, \% & 53.3 & 68.4 & 61.7 & 57.2 & 50.6 \\
\hline \multicolumn{6}{|l|}{ Socioeconomic and lifestyle factors } \\
\hline Urban region, \% & 53.7 & 48.4 & 46.3 & 37.2 & 32.5 \\
\hline$\geq 9$ years of education, $\%$ & 17.8 & 23.4 & 25.4 & 21.6 & 17.1 \\
\hline Household income $\geq ¥ 35$ 000/year, \% & 15.3 & 20.8 & 21.1 & 17.4 & 14.3 \\
\hline \multicolumn{6}{|l|}{ Ever regular smoking, \% } \\
\hline Male & 69.3 & 67.9 & 65.9 & 67.3 & 67.7 \\
\hline Female & 3.5 & 2.7 & 2.5 & 2.5 & 2.5 \\
\hline \multicolumn{6}{|l|}{ Weekly drinking, \% } \\
\hline Male & 30.7 & 34.9 & 34.4 & 34.9 & 34.5 \\
\hline Female & 1.9 & 2.0 & 2.1 & 2.3 & 2.4 \\
\hline Total physical activity (SD), MET-hours/day & $5.9(2.5)$ & $11.8(1.6)$ & $17.8(2.2)$ & $27.1(3.3)$ & $42.1(8.3)$ \\
\hline Sedentary leisure time (SD), hours/day & $3.6(1.8)$ & $3.3(1.5)$ & $3.0(1.4)$ & $2.7(1.4)$ & $2.6(1.3)$ \\
\hline \multicolumn{6}{|l|}{ Blood pressure and anthropometry } \\
\hline $\mathrm{SBP}(\mathrm{SD}), \mathrm{mm} \mathrm{Hg}$ & $131.8(23.2)$ & $131.4(22.0)$ & $130.7(20.7)$ & $130.3(20.2)$ & $129.3(19.4)$ \\
\hline $\mathrm{RPG}(\mathrm{SD}), \mathrm{mmol} / \mathrm{L}$ & $6.2(2.9)$ & $6.1(2.5)$ & $6.0(2.2)$ & $6.0(2.0)$ & $5.9(1.8)$ \\
\hline BMI (SD), kg/m² & $23.9(3.6)$ & $23.8(3.5)$ & $23.7(3.3)$ & $23.4(3.2)$ & $23.3(3.1)$ \\
\hline Waist circumference (SD), cm & $81.2(10.3)$ & $80.9(9.8)$ & $80.3(9.7)$ & $79.5(9.4)$ & $78.9(9.0)$ \\
\hline Hip circumference (SD), cm & $91.3(7.5)$ & $91.3(7.1)$ & $91.0(6.8)$ & $90.5(6.4)$ & $90.0(6.1)$ \\
\hline Waist to hip ratio (SD) & $0.89(0.07)$ & $0.89(0.07)$ & $0.88(0.07)$ & $0.88(0.07)$ & $0.87(0.07)$ \\
\hline Per cent body fat (SD), \% & $28.6(9.0)$ & $28.4(8.6)$ & $27.8(8.1)$ & $27.3(8.0)$ & $26.8(7.8)$ \\
\hline Height (SD), cm & $158.8(8.3)$ & $159.0(8.0)$ & $159.0(8.4)$ & $158.7(8.4)$ & $157.8(7.9)$ \\
\hline \multicolumn{6}{|l|}{ Disease history, \% } \\
\hline Diabetes & 7.8 & 6.3 & 5.3 & 4.4 & 3.6 \\
\hline Coronary heart disease & 3.5 & 3.1 & 2.6 & 2.1 & 1.5 \\
\hline Stroke or TIA & 3.2 & 1.6 & 1.2 & 1.1 & 0.9 \\
\hline Hypertension & 13.9 & 12.8 & 10.8 & 9.5 & 8.6 \\
\hline Family history of diabetes & 4.8 & 5.3 & 5.3 & 5.0 & 4.8 \\
\hline Family history of cancer & 13.7 & 13.8 & 14.0 & 14.3 & 13.6 \\
\hline
\end{tabular}

*Results were standardised by age, sex and region (where appropriate). Values are means unless otherwise stated.

BMI, body mass index; MET, metabolic equivalent of task; RPG, random plasma glucose; SBP, systolic blood pressure; TIA, transient ischaemic attack.

\section{RESULTS}

Baseline characteristics of participants by physical activity Among all 460937 participants included, the mean (SD) age at baseline was $52(10.5)$ years. The mean (SD) total physical activity was 21.2 (13.9) MET-hours/day. Occupational physical activity accounted for a higher proportion of total physical activity in men than in women $(72 \%$ vs $57 \%)$. The patterns of non-occupational physical activity differed by sex and urbanicity (online supplementary table 4). For example, women had almost threefold higher levels of household activity than men, while participants in urban areas had almost tenfold higher levels of leisure-time activity than those in rural areas (mean 1.7 vs 0.2 MET-hours/day). Participants with higher total physical activity were more likely to be men, younger, from rural areas and had lower levels of sedentary leisure time (table 1). They were more likely to have lower levels of systolic blood pressure, random plasma glucose and adiposity, and less likely to have diabetes, cardiovascular disease and hypertension.

\section{Associations of total physical activity with hepatobiliary diseases}

For liver diseases, total physical activity showed inverse associations with hospitalised non-alcoholic fatty liver disease, viral hepatitis, cirrhosis and liver cancer (figure 1 and online supplementary table 5), similar across these subtypes ( $p$ for heterogeneity 0.13 ). There was no evidence of an association with alcoholic liver disease (HR 0.92 (95\% CI 0.70-1.22)), possibly due to the small number of cases involved. For gallbladder diseases, total physical activity showed inverse associations with gallstone disease, gallbladder cancer and biliary cancer but not with cholecystitis (figure 1 and online supplementary table 5), which were similar for GBTC ( $p$ for heterogeneity 0.34 ) but differed for gallstone disease and cholecystitis ( $\mathrm{p}$ for heterogeneity 0.02 ). Likelihood ratio tests showed evidence of significant non-linear associations for gallstone disease and biliary tract cancer ( $\mathrm{p}$ for non-linearity $=0.01$ and 0.02 ) and no strong evidence of non-linear associations for other diseases ( $p$ for nonlinearity $=0.06-0.77$; online supplementary figure 2 ). Similar associations were observed for intrahepatic bile duct cancer and liver cancer and for extrahepatic bile duct cancer and biliary tract cancer (online supplementary table 6).

\section{Associations of domain-specific physical activity with} hepatobiliary diseases

For domain-specific physical activity, the shape and strength of the inverse associations with specific disease type were similar 
for occupational and total physical activity, whereas there were no clear associations for non-occupational physical activity (online supplementary figures 3-4). When compared with major coronary events (major coronary events; ICD-10: I21-I23 and I20, I24 or I25 fatal only), the threshold above which an inverse association was observed for both total and occupational physical activity was higher for chronic liver disease, liver cancer and GBTC and similar for gallstone disease (figure 2). For nonoccupational physical activity, there were inverse associations with major coronary events and no clear patterns for hepatobiliary cancers and diseases.

As the patterns of physical activity differed in urban and rural areas, the associations of domain-specific physical activity with hepatobiliary diseases were presented in urban and rural areas separately (figure 3 ). We combined chronic liver disease and GBTC in subsequent domain-specific analyses because the associations of physical activity with hepatobiliary diseases did not differ across disease subtypes. Overall the associations with hepatobiliary diseases did not differ between occupational and non-occupational physical activity, except for chronic liver disease and liver cancer in rural areas ( $p$ value for heterogeneity $<0.001$; figure 3 and online supplementary figure 5). For chronic liver disease and liver cancer, there were inverse associations for commuting physical activity, while an inverse association for leisure-time physical activity was only observed for liver cancer. In contrast, there were positive associations for household physical activity in rural areas and no clear associations in urban areas. For gallstone disease, inverse trends were observed across four domains. For GBTC, there were inverse trends for occupational and commuting physical activity but no clear associations for leisure-time and household physical activity, probably because of the small number of events. The associations of domain-specific physical activity with hepatobiliary diseases did not differ between urban and rural areas, except for household physical activity with liver cancer and gallstone disease (online supplementary figure 5).

\section{Subgroup and sensitivity analyses}

Overall the associations of physical activity with hepatobiliary disease did not differ by sex except for occupational physical activity and GBTC ( $p$ value for heterogeneity 0.03; online supplementary figure 6). For other population subgroups, the associations of physical activity with hepatobiliary diseases were consistent across population subgroups, with the exception of chronic liver disease by education and smoking (online supplementary figures 7-8). Of note, the associations of physical activity with hepatobiliary diseases did not differ by sedentary leisure time and work activity level. The associations for physical activity with hepatobiliary diseases and cancers were largely unaltered when excluding the first 5 years of follow-up and participants with chronic diseases or poor self-rated health at baseline (online supplementary table 7 and online supplementary figure 9). To explore potential bias, we selected accidental death (ICD-10: V01-X59, excluding cycling accidents
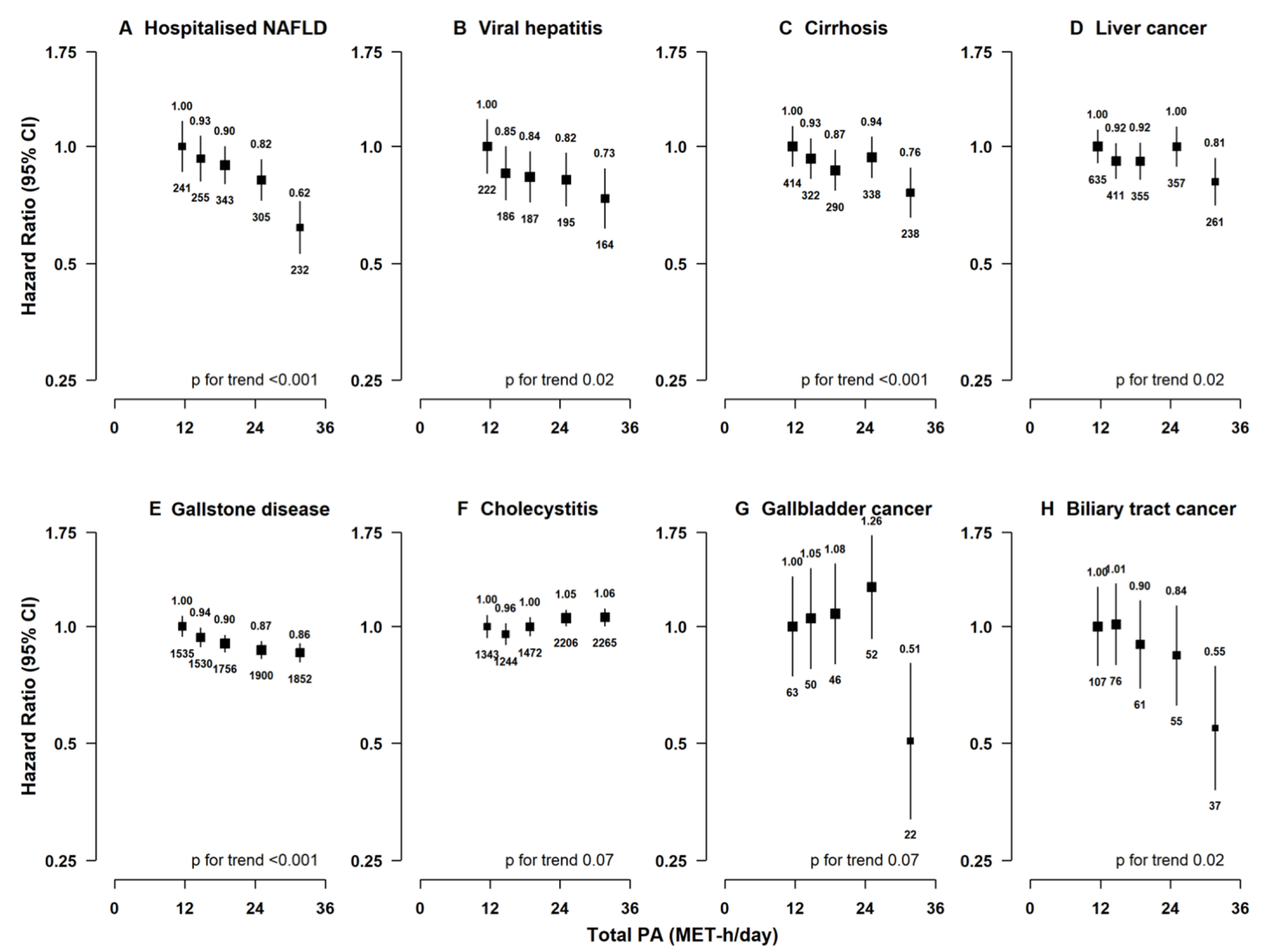

Figure 1 Associations of total physical activity with risk of hepatobiliary diseases. Models were stratified by sex and region, and adjusted for age at baseline, education, household income, smoking, alcohol, self-rated health, diabetes, cardiovascular disease, respiratory disease, rheumatoid arthritis and sedentary leisure time. Time since birth was used as the underlying time scale with delayed entry at age at baseline. HRs are plotted against the mean level in each category of physical activity. Log scale is used for the y axis. The squares represent $\mathrm{HR}$ and the vertical lines represent $95 \% \mathrm{Cl}$. The area of the squares is inversely proportional to the variance of the log HR. The numbers above the vertical lines are point estimates for HR, and the numbers below the lines are the number of events. MET, metabolic equivalent of task; NAFLD, non-alcoholic fatty liver disease; PA, physical activity. 
A Major vascular events vs liver diseases
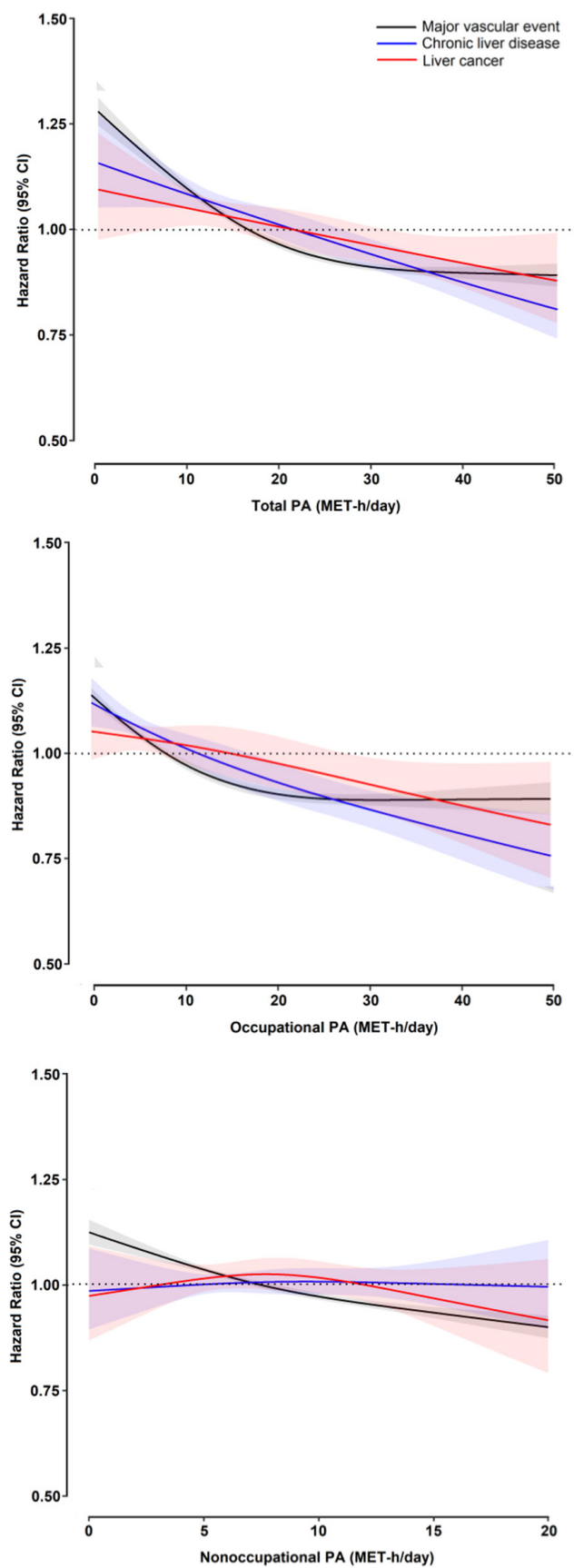

B Major vascular events vs gallbladder diseases
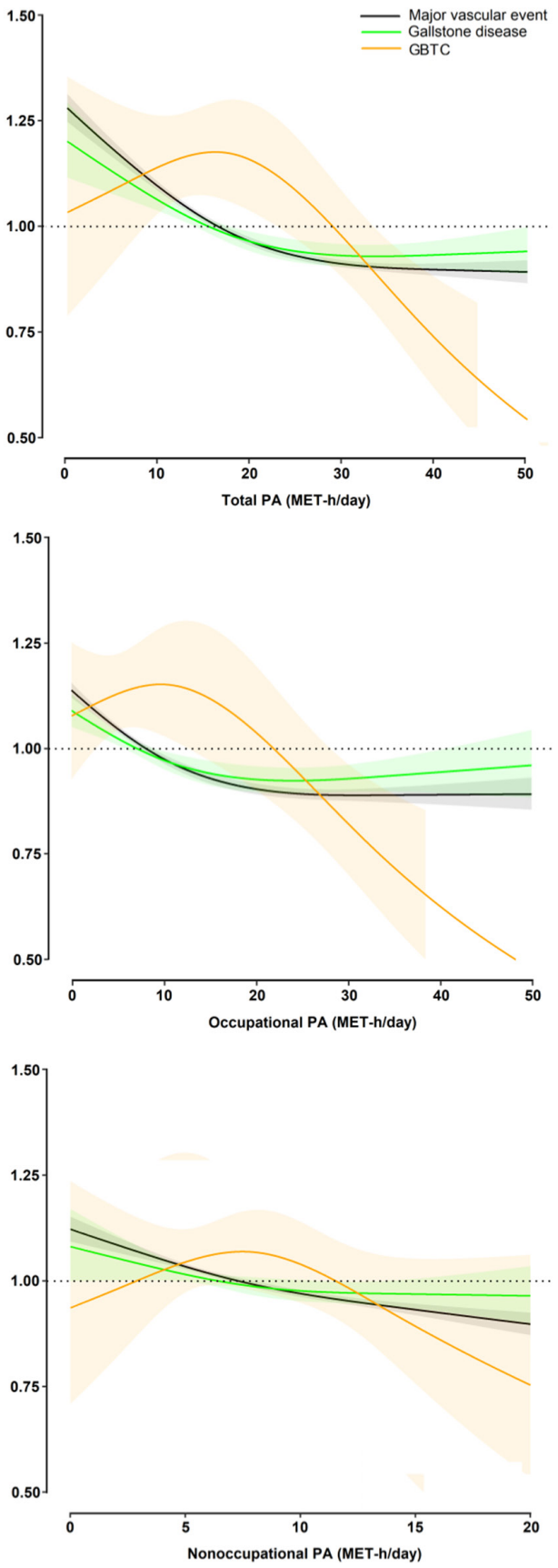

Figure 2 Associations of physical activity with risk of major coronary events and hepatobiliary diseases. Panel A denotes liver diseases and panel B denotes gallbladder diseases. Restricted cubic splines were calculated using three fixed knots at 10\%, 50\% and $90 \%$ quintiles. figure 1 Major coronary events denote major adverse cardiovascular events (ICD-10: I21-I23 from any cause; I20, I24 or I25 only when fatal). Participants with history of cardiovascular diseases were excluded from the analysis of physical activity and major coronary events. GBTC, gallbladder and biliary tract cancer; ICD, International Classification of Diseases; MET, metabolic equivalent of task; PA, physical activity.

(V10-19) and intentional self-harm (X60-84)) as a negative control outcome, which had a similar confounding structure as observed with liver cancer (online supplementary table 8). As shown in online supplementary table 9 and online supplementary figure 10, there were no associations for total, occupational and non-occupational physical activity.

For chronic liver disease, the inverse association for total physical activity attenuated by $37.4 \%$ with additional adjustment for waist circumference (95\% CI $18.6 \%$ to $89.8 \%$ ), while additional adjustment for diabetes changed little of the inverse association
(7.2\%, 95\% CI 2.5\% to 20.6\%; figure 4). The attenuation was greater in men than in women (waist circumference: $47.1 \%$ and $21.5 \%$; diabetes: $8.2 \%$ and $2.2 \%$; online supplementary table 10). For gallbladder disease, the inverse association for total physical activity attenuated by $50.2 \%$ with additional adjustment for waist circumference $(50.2 \%(29.3 \%-80.8 \%))$, and there was little change when additionally adjusting for diabetes $(5.0 \%(2.2 \%-10.3 \%))$. The decrease was similar by sex (online supplementary table 10). In contrast, additional adjustment for waist circumference or diabetes altered little of the inverse 
associations of total physical activity with liver cancer or GBTC (figure 4).

\section{Meta-analyses of CKB with previous prospective studies on} hepatobiliary cancers

Total physical activity showed inverse associations with liver, gallbladder and extrahepatic biliary tract cancers (figure 5). The HRs comparing the top and bottom categories of total physical activity were 0.71 (95\% CI 0.59 to 0.87 ) for liver cancer, 0.55 (95\% CI 0.38 to 0.80 ) for gallbladder cancer and 0.66 (95\% CI 0.48 to 0.92 ) for extrahepatic bile duct cancer, with little between-study heterogeneity $\left(\mathrm{I}^{2}=47.9 \%, \mathrm{I}^{2}=0 \%\right.$ and $\mathrm{I}^{2}=0 \%$, respectively). For liver cancer subtypes, there was an inverse association for hepatocellular carcinoma and no association for intrahepatic bile duct cancer (0.57 (95\% CI 0.46 to 0.70$)$ and 0.92 (95\% CI 0.64 to 1.31$)$ ), with little between-study heterogeneity $\left(\mathrm{I}^{2}=0\right)$. For leisure-time physical activity, there was an inverse association for liver cancer $(0.83,95 \%$ CI 0.75 to 0.91$)$ and no association for gallbladder cancer $(0.86,95 \%$ CI 0.59 to $1.27)$, with moderate between-study heterogeneity $\left(\mathrm{I}^{2}=40.3 \%\right.$ and $\left.\mathrm{I}^{2}=52.9 \%\right)$.

\section{DISCUSSION}

In this Chinese population, total physical activity was inversely associated with several major subtypes of hepatobiliary diseases. The associations for occupational physical activity mirrored those with total physical activity. For non-occupational physical activity, the associations with hepatobiliary diseases differed by domain, possibly reflecting residual confounding. The inverse associations of total physical activity attenuated by $\sim 40 \%$ for chronic liver disease and $\sim 50 \%$ for gallstone disease when we also adjusted for central adiposity. Our main results remained largely unchanged when we excluded (1) the first 5 years of follow-up and (2) participants with chronic diseases or poor selfrated health at study baseline.

\section{Extending the previous findings in Western populations}

Prospective cohort studies in Western populations reported an inverse association for hepatocellular carcinoma but no associations for other subtypes, including intrahepatic bile duct cancer, extrahepatic bile duct cancer and gallbladder cancer. ${ }^{11} 12$ However, these studies involved fewer than 800 hepatobiliary cancer cases and may have limited statistical power. In this CKB population, there were inverse associations between total physical activity and liver cancer and GBTC.

When meta-analysed with previous cohort studies, there were inverse associations for gallbladder cancer and extrahepatic bile duct cancer. For leisure-time physical activity, the inverse association in $\mathrm{CKB}$ is consistent with previous studies in Western and East Asian populations, ${ }^{13}$ 33-37 including a cohort study of 416175 participants and 924 liver cancer cases in Taiwan (HR comparing $\geq 7.5$ MET-hours/day vs none: $0.83(0.75-0.91)$ in CKB vs 0.82 (0.69-0.98) in that study). ${ }^{34}$ For gallbladder cancer, a pooled analysis of 12 cohort studies in the USA and Europe

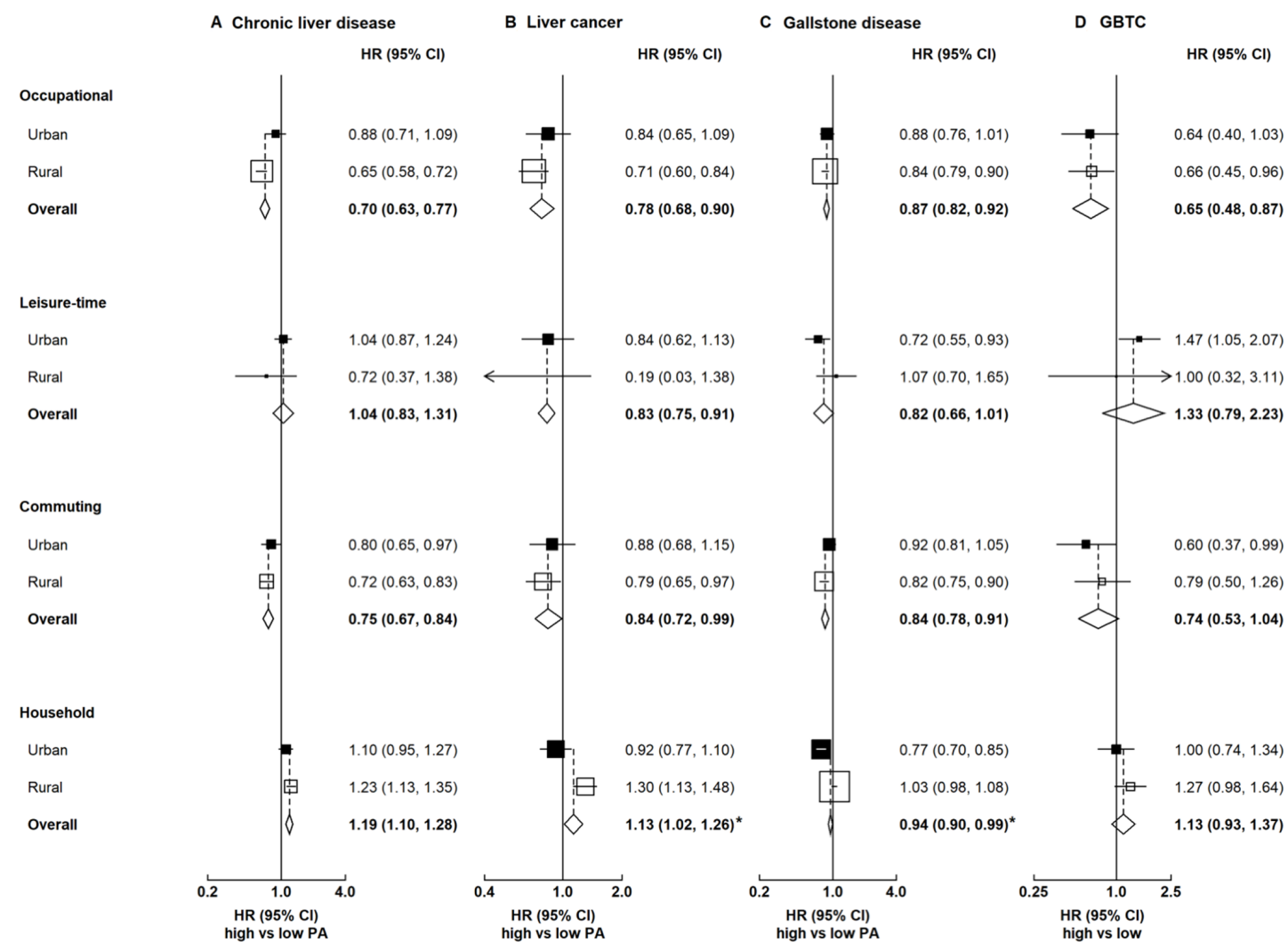

Figure 3 Associations of domain-specific physical activity with hepatobiliary diseases. Boxes represent the HR associated with hepatobiliary disease comparing high and low categories of physical activity, with the size of the box inversely proportional to the variance of the log HR. Diamonds represent summary HR in both urban and rural areas. Asterisk $\left(^{*}\right)$ denotes heterogeneity by urbanicity ( $p$ value for heterogeneity $<0.05$ ). The amount of occupational and non-occupational physical activity was categorised by splitting at quintiles, while the amount of commuting and household physical activity was categorised by splitting at tertiles. In the analysis by urbanicity, region-specific cut-off points were used. For leisure-time physical activity, the HR was comparing $\geq 7.5 \mathrm{MET}$-hours/day (recommended by the 2010 WHO guidelines ${ }^{62}$ ) with none. GBTC, gallbladder and biliary tract cancer; MET, metabolic equivalent of task; PA, physical activity. 


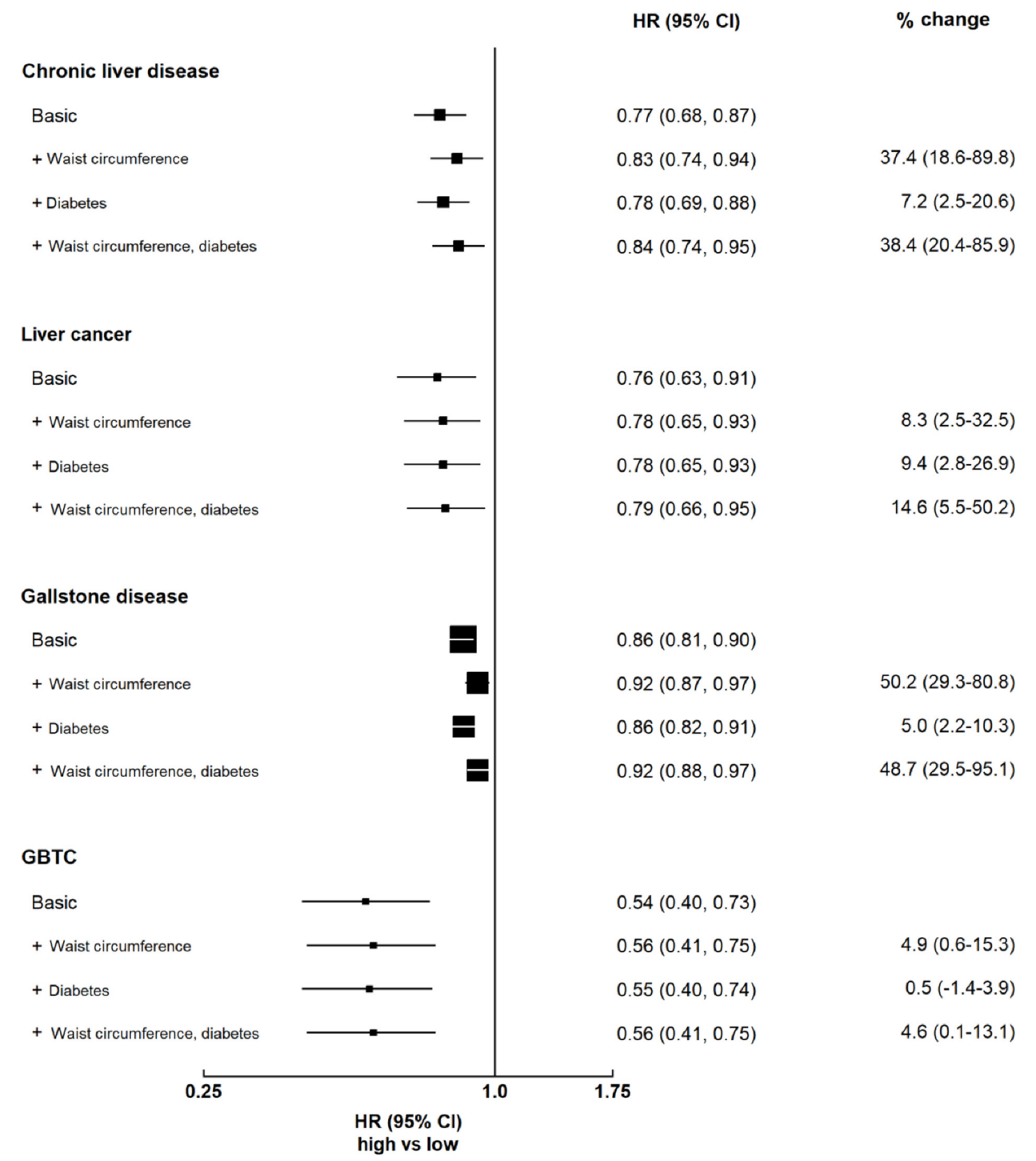

Figure 4 Per cent change in the association of total physical activity with hepatobiliary diseases with additional adjustment for adiposity and diabetes. Boxes represent the HR associated with hepatobiliary cancer comparing high versus low categories of physical activity, with the size of the box inversely proportional to the variance of the log HR. Models were stratified by sex and region, and adjusted for age at baseline, education, household income, smoking, alcohol, self-rated health, diabetes, cardiovascular disease, respiratory disease, rheumatoid arthritis and sedentary leisure time. For chronic liver disease and liver cancer, the first 5 years of follow-up were excluded. GBTC, gallbladder and biliary tract cancer.

with 382 cases reported an inverse association. ${ }^{13}$ However, no association was observed in CKB probably because of the narrower range of leisure-time physical activity (median 0.1 MET-hours/day in CKB vs 8-11 MET-hours/day in the pooled analysis). ${ }^{13}$

For non-alcoholic fatty liver disease, a meta-analysis of six cohort studies with 32657 cases showed a 21\% lower risk comparing high and low categories of physical activity, with similar associations for total and leisure-time physical activities. ${ }^{14}$ In $\mathrm{CKB}$, the finding for total physical activity was consistent with previous studies, whereas there was no clear association for leisure-time physical activity (online supplementary figure 11). A previous randomised controlled trial in Chinese people showed that moderate to vigorous exercise was associated with lower risk of non-alcoholic fatty liver disease. ${ }^{38}$ Therefore, the null association might be due to the low intensity of leisure-time physical activity in CKB. For gallbladder diseases, a meta-analysis of eight cohort studies and 6197 cases reported inverse associations for total and leisure-time physical activities, ${ }^{15}$ consistent with findings in $\mathrm{CKB}$ (online supplementary figure 11). In $\mathrm{CKB}$, we found an inverse association for gallstone disease and no association for cholecystitis ( $\mathrm{p}$ for heterogeneity 0.02 ), consistent with previous studies showing stronger associations of other metabolic risk factors with gallstone disease than cholecystitis. ${ }^{939}$

\section{Associations in rural and urban regions}

In our CKB population, we report heterogeneity in the associations of domain-specific physical activity with chronic liver disease and liver cancer in rural areas. The positive associations of household physical activity in rural areas might reflect confounding by infections.

$\mathrm{HBV}$ is a major risk factor for liver cancer and chronic liver disease, ${ }^{3}$ and household contact (contaminated blood or body fluids) is a major mode of transmission. ${ }^{40}$ In CKB, household physical activity was directly calculated from household hours (no information on types collected) and might be an indicator of household contact. Analysis of the China Nutritional Health 
A Total PA

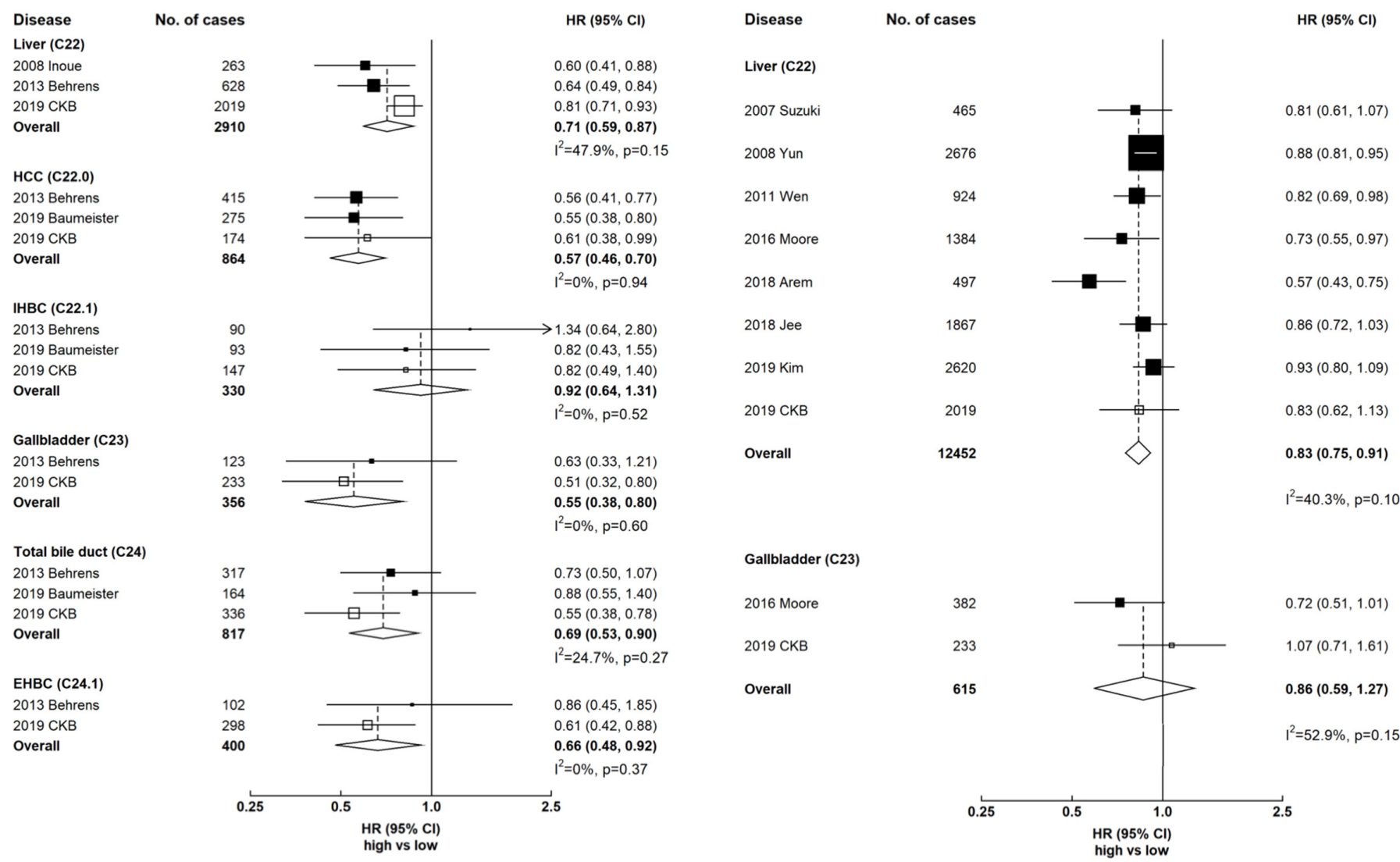

Figure 5 Meta-analysis of prospective studies on physical activity and hepatobiliary cancers. Panel A denotes total physical activity and panel $B$ denotes leisure physical activity. Boxes represent the HR associated with hepatobiliary cancer comparing high versus low categories of physical activity, with the size of the box inversely proportional to the variance of the log HR. Open boxes represent previously published studies, and the black boxes represent CKB estimates. Diamonds represent summary HR for overall. Within categories HRs are ordered according to their year of publication. Estimates and $95 \% \mathrm{Cl}$ of the summary HR are in bold. References for previous studies: Suzuki 2007, PMID 18260704; Inoue 2008, PMID 18599492; Behrens 2013, PMID 23354983; Baumeister 2019, PMID 30582978; Yun 2008, PMID 19077256; Wen 2011, PMID 21846575; Moore 2016, PMID 27183032; Arem 2018, PMID 29533015; Jee 2018, PMID 29914427; Kim 2019, PMID 30872551. CKB, China Kadoorie Biobank; EHBC, extrahepatic bile duct cancer; HCC, hepatocellular carcinoma; IHBC, intrahepatic bile duct cancer; PA, physical activity.

Survey showed that the proportion of household physical activity involving direct household contact was much higher in rural than in urban areas $(66.9 \%$ vs $31.9 \%) .{ }^{41}$ In contrast, no associations were observed for household physical activity and gallstone disease and GBTC, which were unlikely to be associated with infectious agents. ${ }^{5}$ In addition, when accidental death was used as a negative control outcome, there were no overall associations of total, occupational and non-occupational physical activity with risk of accidental death (online supplementary table 9 and online supplementary figure 10). This suggests that potential confounding and bias from other sources are likely to be minimal.

\section{On causality}

Although the current study findings cannot be used to establish causality, the inverse associations of physical activity with hepatobiliary diseases may not be fully explained by residual confounding and reverse causality. Early prospective studies conducted in Western populations reported inverse associations of occupational physical activity with major chronic diseases, whereas more recent studies have shown inconsistent associations. $^{42} 43$ This change over time might reflect confounding by socioeconomic status. In CKB, we adjusted for education and household income, which have been shown to be good indicators of socioeconomic status in this Chinese population. ${ }^{44} \mathrm{We}$ further showed that the inverse associations were consistent in different birth cohorts in CKB (online supplementary table 11). In addition, the EPIC study, a cohort study of European adults conducted over a similar period and with similar age distribution as $\mathrm{CKB}$, showed that occupational physical activity was associated with lower risks of metabolic diseases, including colorectal cancer and liver cancer. ${ }^{1245}$

Taken together, the consistency of the results over time and in diverse populations suggests that the inverse associations for occupational physical activity are likely to be real. A sensitivity analysis showed that an unmeasured confounder would have to be associated with physical activity and hepatobiliary diseases by a risk ratio of 1.6-3.0, above and beyond the measured confounders, to explain away the observed HR (online supplementary table 12), but weaker confounding could not do so. ${ }^{46}$ In addition, the associations remained for hepatobiliary diseases when excluding the first 5 years of follow-up or participants with chronic diseases or poor self-rated health at baseline, suggesting that the inverse associations were not affected by reverse causality. ${ }^{47}$ 
The role of central adiposity

We found that additional adjustment for central adiposity partially attenuated the inverse associations of physical activity with chronic liver disease and gallstone disease. In line with our observation, a randomised controlled trial among patients with non-alcoholic fatty liver disease showed that vigorous and moderate exercise reduced intrahepatic triglyceride content and the effect was largely mediated by weight loss. ${ }^{38}$ For liver cancer, the EPIC study showed that waist circumference explained $40 \%$ of the inverse associations for total physical activity, ${ }^{12}$ whereas waist circumference explained little of the inverse association in CKB. This is possibly because a much smaller proportion of liver cancer is attributable to metabolic risk factors in China than in Western countries. ${ }^{18}$

\section{Mechanisms: how might physical activity affect the hepatobiliary system?}

There are several potential mechanisms why physical activity is associated with lower risk of hepatobiliary cancers and diseases. ${ }^{1748}$ First, higher physical activity is associated with lower risks of adiposity, hyperinsulinaemia and diabetes, ${ }^{49} 50$ which are associated with hepatobiliary cancers and diseases. ${ }^{39} 51-53$ Second, physical activity is associated with lower levels of systemic inflammation by altering proinflammatory and antiinflammatory factors or adipokines (eg, C-reactive protein, adiponectin and interleukin-6), ${ }^{54}$ which are associated with higher risk of hepatobiliary cancers and diseases. ${ }^{56} 57$ Third, physical activity may have inverse associations by influencing the number and function of natural killer cells. ${ }^{58} 59$ Fourth, physical activity may influence risk of gallbladder disease by modulating gallbladder motility and increasing vagal tone and gastrointestinal transit time,${ }^{60}$ which can promote bile stasis and crystal formation and decrease cholesterol resorption, leading to gallstones. $^{39}$

\section{Strengths and limitations}

The strengths of this CKB study include its prospective design, a large and diverse study population, and data on both occupational and non-occupational physical activity. We adjusted extensively for risk factors for hepatobiliary diseases.

We note at least four limitations. First, our study relied on hospital records to capture hepatobiliary diseases, which may have resulted in underestimation of the disease rates, particularly for non-alcoholic fatty liver disease, which is often 'silent'. However, our risk estimates are unlikely to be greatly biased because (1) we ascertained all non-alcoholic fatty liver disease cases diagnosed between 2013 and 2015 and showed that 93\% of all cases were diagnosed by ultrasound or CT; (2) our risk estimates agreed with previous studies of ultrasound-detected non-alcoholic fatty liver disease and symptomatic gallstone disease and our risk estimates were consistent across population subgroups (online supplementary figures 7, 8 and 12); (3) hospitalised non-alcoholic fatty liver disease and alcoholic liver disease have been shown to be valid in previous CKB reports on adiposity and diabetes; ${ }^{53}{ }^{61}$ and (4) the misclassification is unlikely to differ by level of physical activity or other variables, which suggests that the association would be biased towards the null.

Second, the use of self-report may underestimate prevalent liver diseases at baseline, particularly for alcoholic liver disease and non-alcoholic fatty liver disease. For non-alcoholic fatty liver disease, we showed similar associations when excluding participants with elevated alanine transaminase $(\geq 33 \mathrm{U} / \mathrm{L}$ for men and $\geq 25 \mathrm{U} / \mathrm{L}$ for women) in a nested case-control study of $\sim 18000$ participants with blood biochemistry data (online supplementary table 13).

Third, although similar associations were observed when excluding the first 5 years of follow-up and when excluding participants with chronic diseases or poor self-rated health at baseline, reverse causality cannot be fully addressed because of the long latency period of cancer. Fourth, residual confounding may still exist due to unknown or unmeasured (eg, socioeconomic status, infections, inflammation and lipids) factors.

In summary, this large prospective study provides new evidence that higher levels of total physical activity, particularly occupational physical activity, were inversely associated with risk of major hepatobiliary diseases in Chinese adults, including non-alcoholic fatty liver disease, cirrhosis and cancer. The associations of total physical activity were consistent with previous prospective cohort studies conducted mostly in Western countries. For non-occupational physical activity (particularly leisuretime physical activity), more evidence is needed in populations with a diverse range of activities.

\section{What are the findings?}

In this Chinese population, total physical activity was inversely associated with a diverse range of hepatobiliary diseases, with similar patterns seen for occupational physical activity.

- The associations for non-occupational physical activity differed by disease subtype, with an inverse association for leisure-time physical activity with liver cancer and an inverse trend for gallstone disease.

\section{How might it impact on clinical practice in the future?}

- The benefits of higher levels of total physical activity are independent of lifestyle factors, including smoking, alcohol and sedentary leisure time, and are generally consistent across subtypes of hepatobiliary diseases.

- We identified that more evidence is needed in populations with a diverse range of activities to understand the effects of non-occupational physical activity on hepatobiliary health.

\section{Author affiliations}

'Department of Epidemiology and Biostatistics, School of Public Health, Peking University Health Science Center, Beijing, China

${ }^{2}$ Clinical Trial Service Unit \& Epidemiological Studies Unit (CTSU), Nuffield Department of Population Health, Big Data Institute Building, Roosevelt Drive, University of Oxford, Oxford, UK

${ }^{3}$ Medical Research Council Population Health Research Unit (MRC PHRU), Nuffield Department of Population Health, University of Oxford, Oxford, UK

${ }^{4}$ Chinese Academy of Medical Sciences, Beijing, China

${ }^{5}$ National Institute for Health Research Oxford Biomedical Research Centre, Oxford University Hospital, Oxford, UK

${ }^{6}$ Zhejiang Center for Disease Prevention and Control, Hangzhou, China

${ }^{7}$ Liuzhou Chinese Medicine Hospital, Liuzhou, China

${ }^{8}$ National Center for Food Safety Risk Assessment, Beijing, China

Acknowledgements The chief acknowledgement is to the participants, the project staff, and the China National Center for Disease Control and Prevention and its regional offices for access to death and disease registries. The Chinese National Health Insurance scheme provides electronic linkage to all hospital admission data.

Contributors $\mathrm{LL}$ and $\mathrm{ZC}$ had full access to the data. YP, JL, CK, MVH and ZC conducted data analysis and are responsible for accuracy of the results and the decision to submit for publication. All authors were involved in study design, conduct, long-term follow-up, review and coding of disease events, interpretation of the results, or writing the report. 
Funding Baseline survey: Kadoorie Charitable Foundation, Hong Kong. Long-term continuation: UK Wellcome Trust (088158/Z/09/Z, 104085/Z/14/Z), Chinese Ministry of Science and Technology (2011BAI09B01, 2012-14), and Chinese National Natura Science Foundation (81390541). The British Heart Foundation, UK Medical Research Council and Cancer Research UK provide core funding to the Oxford CTSU. MVH is supported by a British Heart Foundation Intermediate Clinical Research Fellowship (FS/18/23/33512). MVH and DB are supported by the National Institute for Health Research Oxford Biomedical Research Centre. YP acknowledges support from the China Postdoctoral Science Foundation (2019TQ0008). The funders had no role in the collection, analysis or interpretation of data, in the writing of the report, or in the decision to submit the article for publication.

Disclaimer The views expressed are those of the authors and not necessarily those of the NHS, the NIHR or the Department of Health and Social Care.

\section{Competing interests None declared.}

Patient and public involvement Patients and/or the public were not involved in the design, or conduct, or reporting, or dissemination plans of this research.

Patient consent for publication Not required.

Ethics approval The project was approved by the ethical committee and research council of the Chinese Center for Disease Control and Prevention (Beijing, China, 005/2004) and the Oxford Tropical Research Ethics Committee at the University of Oxford (UK, 025-04), and informed written consent was obtained from each participant.

Provenance and peer review Not commissioned; internally peer reviewed.

Data availability statement Data are available upon reasonable request. The China Kadoorie Biobank (CKB) is a global resource for the investigation of lifestyle, environmental, blood biochemical and genetic factors as determinants of common diseases. The CKB study group is committed to making the cohort data available to the scientific community in China, the UK and worldwide to advance knowledge about the causes, prevention and treatment of disease. For detailed information on what is currently available to open access users, visit: http://www. ckbiobank.org/site/Data+Access. Researchers who are interested in obtaining the raw data from the CKB study that underline this paper should contact ckbaccess@ndph.ox.ac.uk. A research proposal will be requested to ensure that any analysis is performed by bona fide researchers and-where data are not currently available to open access researchers-is restricted to the topic covered in this paper.

Open access This is an open access article distributed in accordance with the Creative Commons Attribution 4.0 Unported (CC BY 4.0) license, which permits others to copy, redistribute, remix, transform and build upon this work for any purpose, provided the original work is properly cited, a link to the licence is given, and indication of whether changes were made. See: https://creativecommons.org/ licenses/by/4.0/

\section{ORCID iD}

Yuanjie Pang http://orcid.org/0000-0002-4826-8861

\section{REFERENCES}

1 GBD 2017 Causes of Death Collaborators. Global, regional, and national agesex-specific mortality for 282 causes of death in 195 countries and territories, 1980-2017: a systematic analysis for the global burden of disease study 2017. Lancet 2018;392:1736-88

2 Zhou M, Wang H, Zhu J, et al. Cause-Specific mortality for 240 causes in China during 1990-2013: a systematic subnational analysis for the global burden of disease study 2013. Lancet 2016;387:251-72.

3 Wang F-S, Fan J-G, Zhang Z, et al. The global burden of liver disease: the major impact of China. Hepatology 2014;60:2099-108.

4 Lammert F, Gurusamy K, Ko CW, et al. Gallstones. Nat Rev Dis Primers 2016;2:16024.

5 Stinton LM, Shaffer EA. Epidemiology of gallbladder disease: cholelithiasis and cancer. Gut Liver 2012;6:172-87.

6 Word Cancer Research Fund/American Insisute for Cancer Research. Continuous update project expert report 2018. diet, nutrition, physical activity and liver cancer. Available: dietandcancerreport.org

7 Word Cancer Research Fund/American Insisute for Cancer Research. Continuous update project expert report 2018. diet, nutrition, physical activity and gallbladder cancer. Available: dietandcancerreport.org

8 Tsochatzis EA, Bosch J, Burroughs AK. Liver cirrhosis. Lancet 2014;383:1749-61.

9 Aune D, Norat T, Leitzmann M, et al. Physical activity and the risk of type 2 diabetes: a systematic review and dose-response meta-analysis. Eur J Epidemiol 2015:30:529-42.

10 Aune D, Vatten LJ, Boffetta P. Tobacco smoking and the risk of gallbladder disease. Eur J Epidemiol 2016;31:643-53.

11 Behrens G, Leitzmann MF. The association between physical activity and renal cancer: systematic review and meta-analysis. Br J Cancer 2013;108:798-811.
12 Baumeister SE, Schlesinger S, Aleksandrova K, et al. Association between physical activity and risk of hepatobiliary cancers: a multinational cohort study. J Hepatol 2019;70:885-92.

13 Moore SC, Lee I-M, Weiderpass E, et al. Association of leisure-time physica activity with risk of 26 types of cancer in 1.44 million adults. JAMA Intern Med 2016;176:816-25.

14 Qiu S, Cai X, Sun Z, et al. Association between physical activity and risk of nonalcoholic fatty liver disease: a meta-analysis. Therap Adv Gastroenterol 2017;10:701-13

15 Aune D, Leitzmann M, Vatten LJ. Physical activity and the risk of gallbladder disease: a systematic review and meta-analysis of cohort studies. J Phys Act Health 2016;13:788-95.

16 Kahn SE, Hull RL, Utzschneider KM. Mechanisms linking obesity to insulin resistance and type 2 diabetes. Nature 2006;444:840-6.

17 Warburton DER, Nicol CW, Bredin SSD. Health benefits of physical activity: the evidence. CMAJ 2006;174:801-9.

18 Global Burden of Disease Liver Cancer Collaboration, Akinyemiju T, Abera S, et al. The burden of primary liver cancer and underlying etiologies from 1990 to 2015 at the global, regional, and national level: results from the global burden of disease study 2015. JAMA Oncol 2017;3:1683-91.

19 Ratib S, West J, Fleming KM. Liver cirrhosis in England-an observational study: are we measuring its burden occurrence correctly? BMJ Open 2017;7:e013752.

20 Beste LA, Leipertz SL, Green PK, et al. Trends in burden of cirrhosis and hepatocellular carcinoma by underlying liver disease in US veterans, 2001-2013. Gastroenterology 2015;149:1471-82.

21 Du H, Bennett D, Li L, et al. Physical activity and sedentary leisure time and their associations with BMI, waist circumference, and percentage body fat in 0.5 million adults: the China Kadoorie Biobank study. Am J Clin Nutr 2013:97:487-96.

22 Hallal PC, Andersen LB, Bull FC, et al. Global physical activity levels: surveillance progress, pitfalls, and prospects. Lancet 2012;380:247-57.

23 Chen Z, Chen J, Collins R, et al. China Kadoorie Biobank of 0.5 million people: survey methods, baseline characteristics and long-term follow-up. Int I Epidemiol 2011:40:1652-66.

24 Wareham NJ, Jakes RW, Rennie KL, et al. Validity and repeatability of the EPIC-Norfolk physical activity questionnaire. Int J Epidemiol 2002:31:168-74.

25 Matthews $C E$, Shu X-O, Yang G, et al. Reproducibility and validity of the Shanghai women's health study physical activity questionnaire. Am J Epidemiol 2003; 158:1114-22.

26 Ainsworth BE, Haskell WL, Herrmann SD, et al. 2011 compendium of physica activities: a second update of codes and Met values. Med Sci Sports Exerc 2011:43:1575-81.

27 Yang G, Rao C, Ma J, et al. Validation of verbal autopsy procedures for adult deaths in China. Int J Epidemiol 2006;35:741-8.

28 Easton DF, Peto J, Babiker AG. Floating absolute risk: an alternative to relative risk in survival and case-control analysis avoiding an arbitrary reference group. Stat Med 1991:10:1025-35

29 MacMahon S, Peto R, Cutler J, et al. Blood pressure, stroke, and coronary heart disease. Part 1, prolonged differences in blood pressure: prospective observational studies corrected for the regression dilution bias. Lancet 1990;335:765-74.

30 Suzuki K. Japan collaborative cohort study for evaluation of cancer. health conditions and mortality in the Japan collaborative cohort study for evaluation of cancer (JACC). Asian Pac J Cancer Prev 2007:8:25-34.

31 Inoue M, Yamamoto S, Kurahashi N, et al. Daily total physical activity level and total cancer risk in men and women: results from a large-scale population-based cohort study in Japan. Am J Epidemiol 2008:168:391-403.

32 Arem H, Loftfield E, Saint-Maurice PF, et al. Physical activity across the lifespan and liver cancer incidence in the NIH-AARP diet and health study cohort. Cancer Med 2018;7:1450-7.

33 Yun YH, Lim MK, Won Y-J, et al. Dietary preference, physical activity, and cancer risk in men: National health insurance Corporation study. BMC Cancer 2008;8:366.

34 Wen CP, Wai JPM, Tsai MK, et al. Minimum amount of physical activity for reduced mortality and extended life expectancy: a prospective cohort study. Lancet 2011;378:1244-53

35 Arem $\mathrm{H}$, Moore SC, Park Y, et al. Physical activity and cancer-specific mortality in the NIH-AARP diet and health study cohort. Int I Cancer 2014;135:423-31.

36 Jee Y, Kim Y, Jee SH, et al. Exercise and cancer mortality in Korean men and women: a prospective cohort study. BMC Public Health 2018;18:761.

37 Kim Y, Sharp S, Hwang S, et al. Exercise and incidence of myocardial infarction, stroke hypertension, type 2 diabetes and site-specific cancers: prospective cohort study of 257854 adults in South Korea. BMJ Open 2019:9:e025590.

38 Zhang H-J, He J, Pan L-L, et al. Effects of moderate and vigorous exercise on nonalcoholic fatty liver disease: a randomized clinical trial. JAMA Intern Med 2016;176:1074-82

39 Aune D, Vatten LJ. Diabetes mellitus and the risk of gallbladder disease: a systematic review and meta-analysis of prospective studies. J Diabetes Complications 2016;30:368-73.

40 Mast EE, Alter MJ, Margolis HS. Strategies to prevent and control hepatitis B and C virus infections: a global perspective. Vaccine 1999;17:1730-3 
41 Monda KL, Adair LS, Zhai F, et al. Longitudinal relationships between occupational and domestic physical activity patterns and body weight in China. Eur J Clin Nutr 2008;62:1318-25.

42 Wahid A, Manek N, Nichols M, et al. Quantifying the association between physical activity and cardiovascular disease and diabetes: a systematic review and metaanalysis. J Am Heart Assoc 2016;5:1-3.

43 Holtermann A, Marott JL, Gyntelberg F, et al. Occupational and leisure time physical activity: risk of all-cause mortality and myocardial infarction in the Copenhagen City heart study. A prospective cohort study. BMJ Open 2012;2:e000556.

44 Pang Y, Kartsonaki C, Guo Y, et al. Socioeconomic status in relation to risks of major gastrointestinal cancers in Chinese adults: a prospective study of 0.5 million people. Cancer Epidemiol Biomarkers Prev 2020;29:823-31.

45 Friedenreich C, Norat T, Steindorf K, et al. Physical activity and risk of colon and recta cancers: the European prospective investigation into cancer and nutrition. Cancer Epidemiol Biomarkers Prev 2006;15:2398-407.

46 VanderWeele TJ, Ding P. Sensitivity analysis in observational research: introducing the E-value. Ann Intern Med 2017;167:268-74.

47 Tarp J, Hansen BH, Fagerland MW, et al. Accelerometer-measured physical activity and sedentary time in a cohort of US adults followed for up to 13 years: the influence of removing early follow-up on associations with mortality. Int J Behav Nutr Phys Act 2020;17:39.

48 McTiernan A. Mechanisms linking physical activity with cancer. Nat Rev Cancer 2008;8:205-11.

49 Mayer-Davis EJ, D'Agostino R, Karter AJ, et al. Intensity and amount of physical activity in relation to insulin sensitivity: the insulin resistance atherosclerosis study. JAMA 1998;279:669-74.

50 Kelley DE, Goodpaster BH. Effects of physical activity on insulin action and glucose tolerance in obesity. Med Sci Sports Exerc 1999;31:S619-23.
51 Kyrgiou M, Kalliala I, Markozannes G, et al. Adiposity and cancer at major anatomical sites: umbrella review of the literature. BMJ 2017;356:477.

52 Giovannucci E, Harlan DM, Archer MC, et al. Diabetes and cancer: a consensus report. Diabetes Care 2010:33:1674-85.

53 Pang Y, Kartsonaki C, Turnbull I, et al. Diabetes, plasma glucose, and incidence of fatty liver, cirrhosis, and liver cancer: a prospective study of 0.5 million people. Hepatology 2018;68:1308-18.

54 Geffken DF, Cushman M, Burke GL, et al. Association between physical activity and markers of inflammation in a healthy elderly population. Am J Epidemiol 2001;153:242-50.

55 Mora S, Lee I-M, Buring JE, et al. Association of physical activity and body mass index with novel and traditional cardiovascular biomarkers in women. JAMA 2006:295:1412-9.

56 Aleksandrova K, Boeing H, Nöthlings $U$, et al. Inflammatory and metabolic biomarkers and risk of liver and biliary tract cancer. Hepatology 2014;60:858-71.

57 Gao B, Tsukamoto H. Inflammation in alcoholic and nonalcoholic fatty liver disease: friend or foe? Gastroenterology 2016;150:1704-9.

58 Woods JA. Physical activity, exercise, and immune function. Brain Behav Immun 2005:19:369-70.

59 Pedersen BK, Ullum H. Nk cell response to physical activity: possible mechanisms of action. Med Sci Sports Exerc 1994;26:140-6.

60 Shephard RJ. Physical activity and the biliary tract in health and disease. Sports Med 2015;45:1295-309.

61 Pang Y, Kartsonaki C, Turnbull I, et al. Adiposity in relation to risks of fatty liver, cirrhosis and liver cancer: a prospective study of 0.5 million Chinese adults. Sci Rep 2019;9:785.

62 World Health Organisation. Global recommendations on physical activity for health. Geneva 2010. 